\title{
New insights to the difference in microbial composition and interspecies interactions between fouling layer and mixed liquor in membrane bioreactor
}

\author{
Revised Supplementary Information \\ Anh Q. Nguyen ${ }^{\mathrm{a}}$, Luong N. Nguyen ${ }^{\mathrm{a}}$, Zhicheng $\mathrm{Xu}^{\mathrm{b}}$, Wenhai Luo ${ }^{\mathrm{b}}$, Long D. Nghiem ${ }^{\mathrm{a}, *}$
}

${ }^{a}$ Centre for Technology in Water and Wastewater, School of Civil and Environmental Engineering, University of Technology Sydney, Ultimo NSW 2007, Australia

${ }^{\mathrm{b}}$ Beijing Key Laboratory of Farmland Soil Pollution Prevention and Remediation, College of Resources and Environmental Sciences, China Agricultural University, Beijing, 100193, China

*Corresponding author:

Long D Nghiem: Centre for Technology in Water and Wastewater, University of Technology Sydney, Ultimo NSW 2007, Australia; E-mail: duclong.nghiem@uts.edu.au 
(A)

(B)
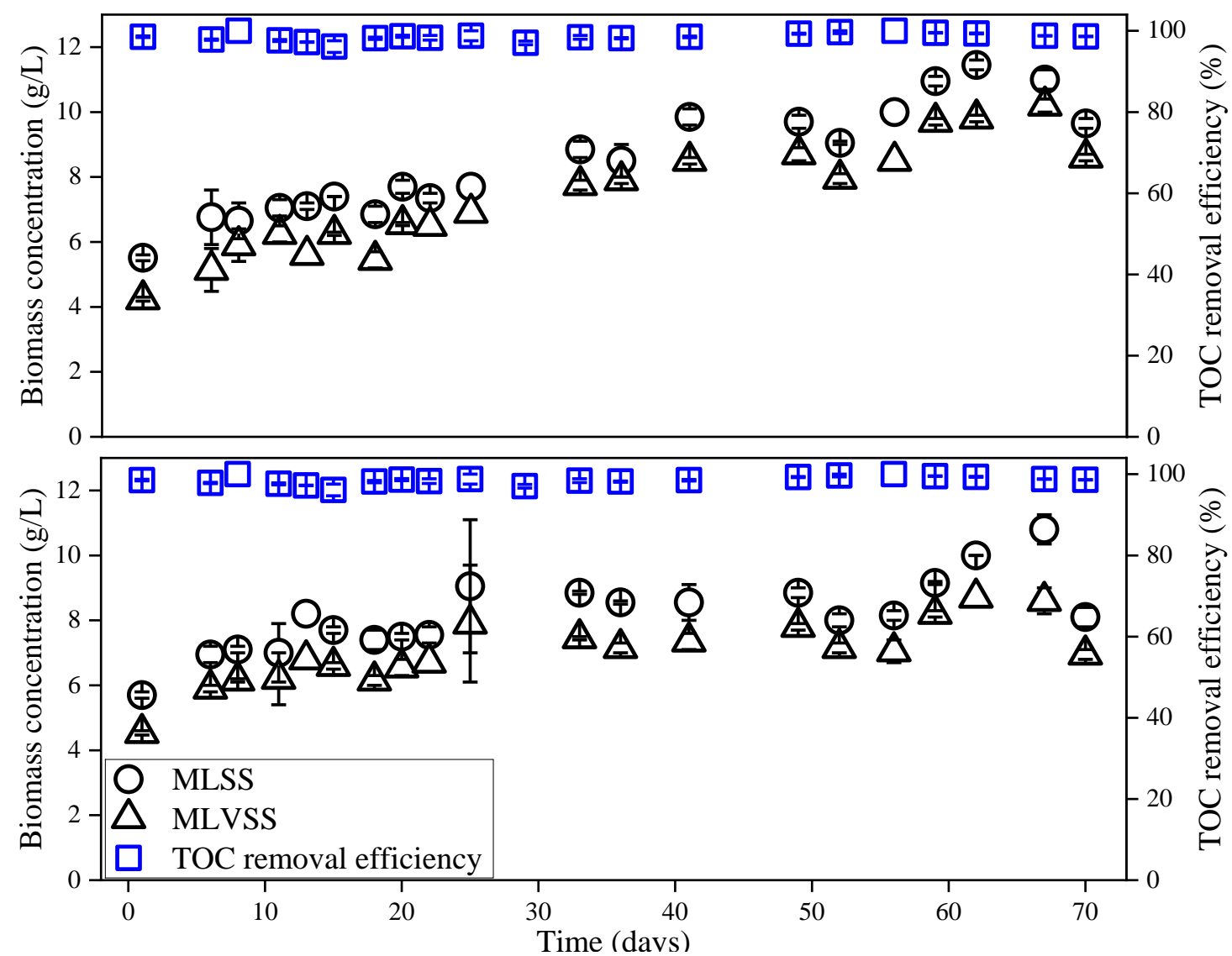

Figure S1. Biomass quality in terms of mixed liquor suspended solids (MLSS), mixed liquor volatile suspended solids (MLVSS), and total organic carbon (TOC) removal efficiency in the two "parent" MBR systems from which the inoculum was taken. 
$15 \mathrm{LMH}$

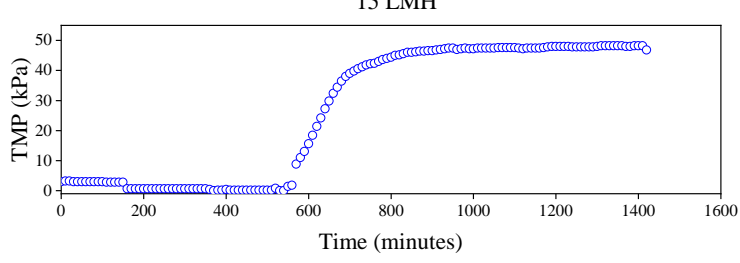

$13 \mathrm{LMH}$

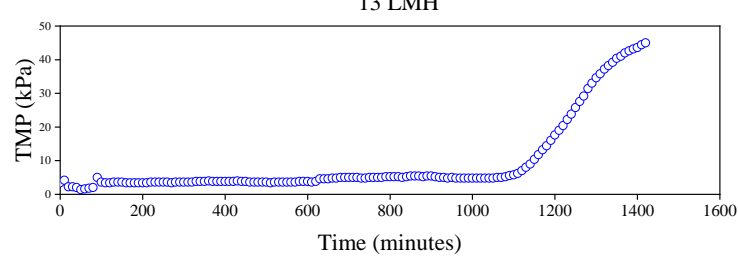

$11 \mathrm{LMH}$

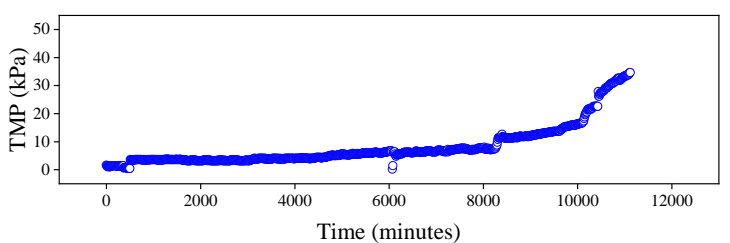

$14 \mathrm{LMH}$

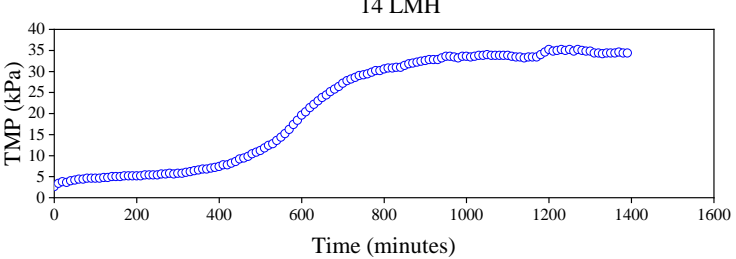

$12 \mathrm{LMH}$

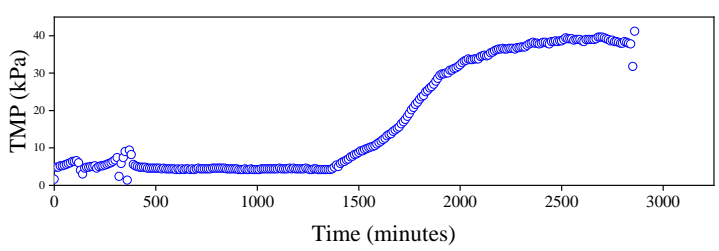

$10 \mathrm{LMH}$

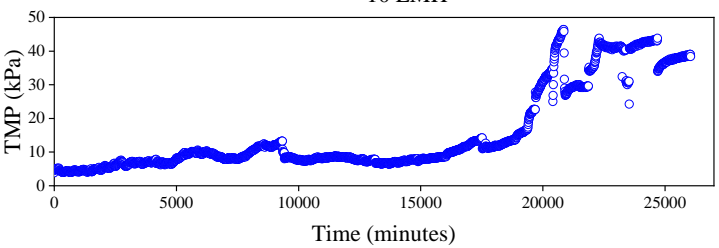

Figure S2. Transmembrane pressure profile of the MBR during preliminary fouling runs in comparison with first biomass collection phases. 
(A)

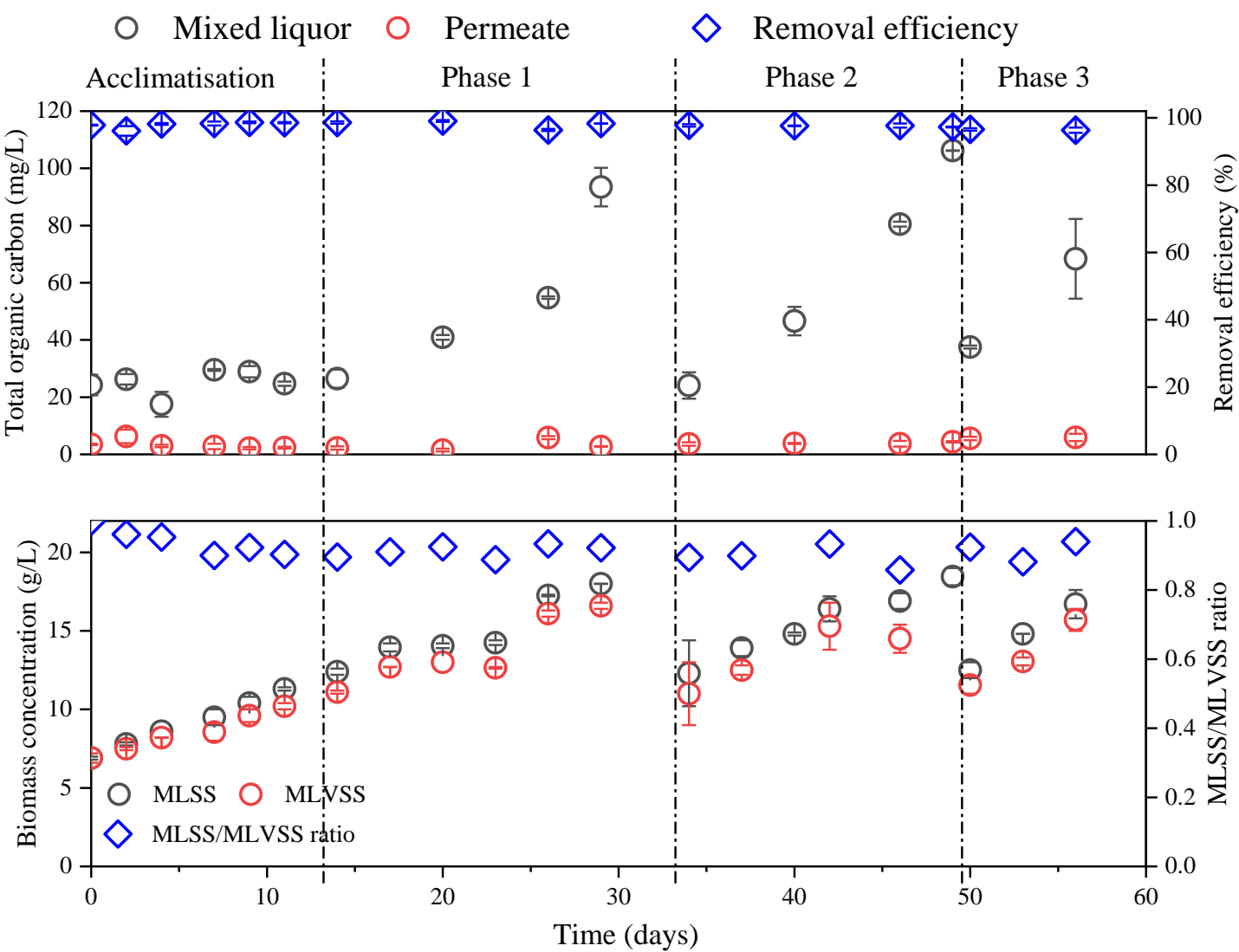

Figure S3. Total organic carbon concentration and removal efficiency (A) and biomass concentration (B) during the experiment. The error bar represents the standard deviation from duplicate samples. 


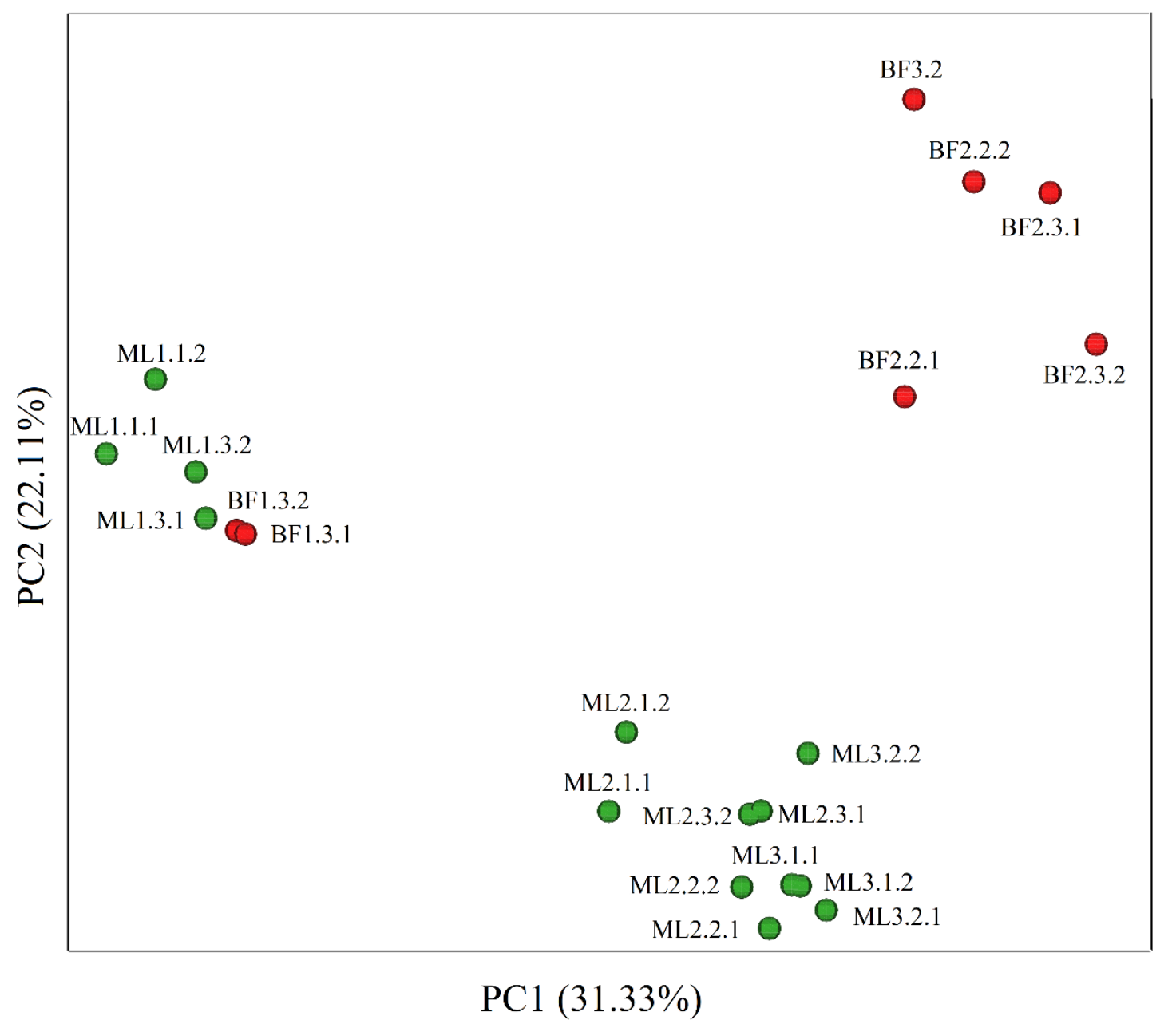

Figure S4. Principal coordinates analysis showing similarity/dissimilarity between mixed liquor (ML) and biofilm (BF) samples microbial community composition. 


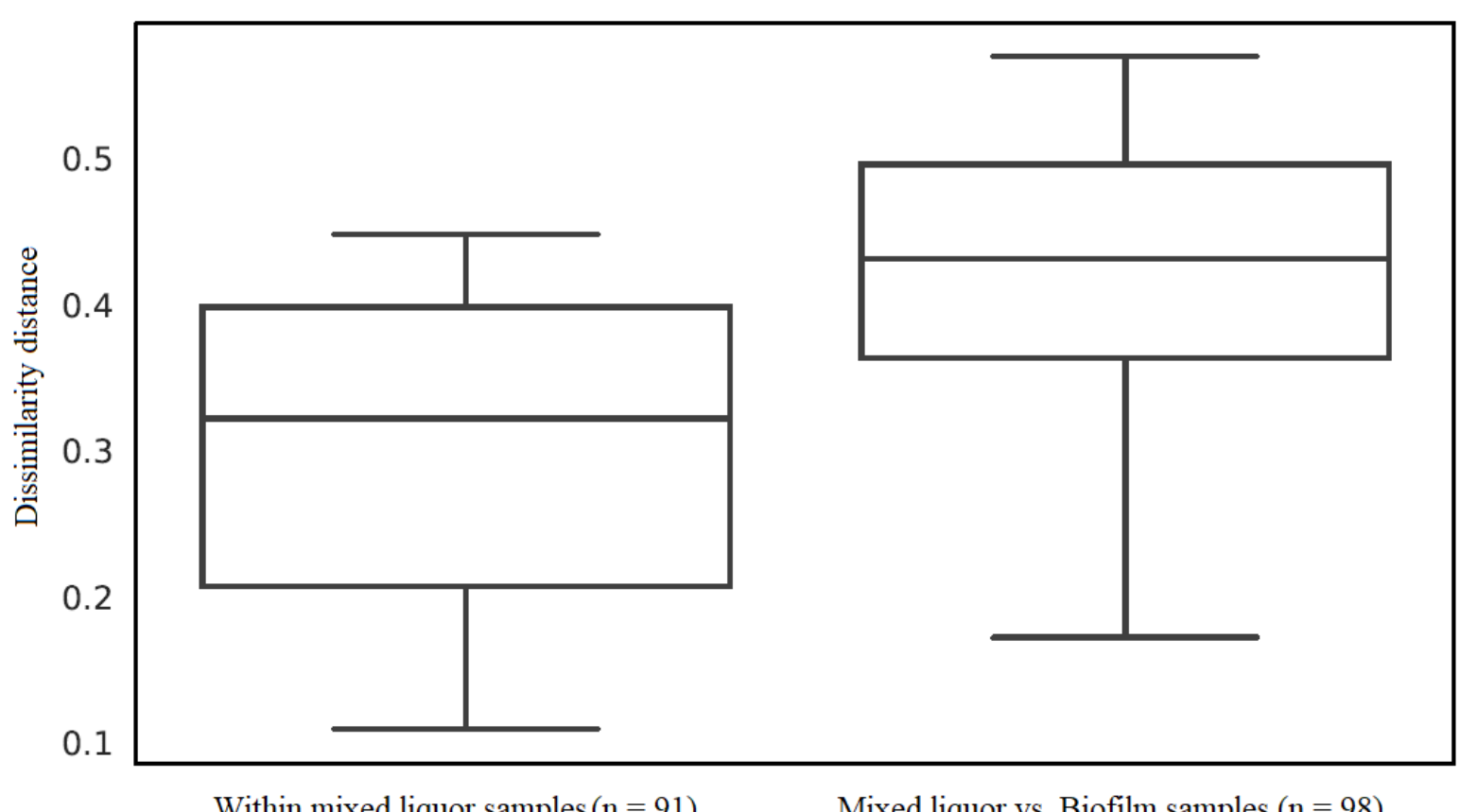

Figure S5. Bray-Curtis dissimilarity distance within mixed liquor samples and between mixed liquor and biofilm samples. The whiskers of the box represent the minimum and maximum values. The bottom and top of the box are the first and third quartiles, respectively, and the line inside the box denotes the median. 

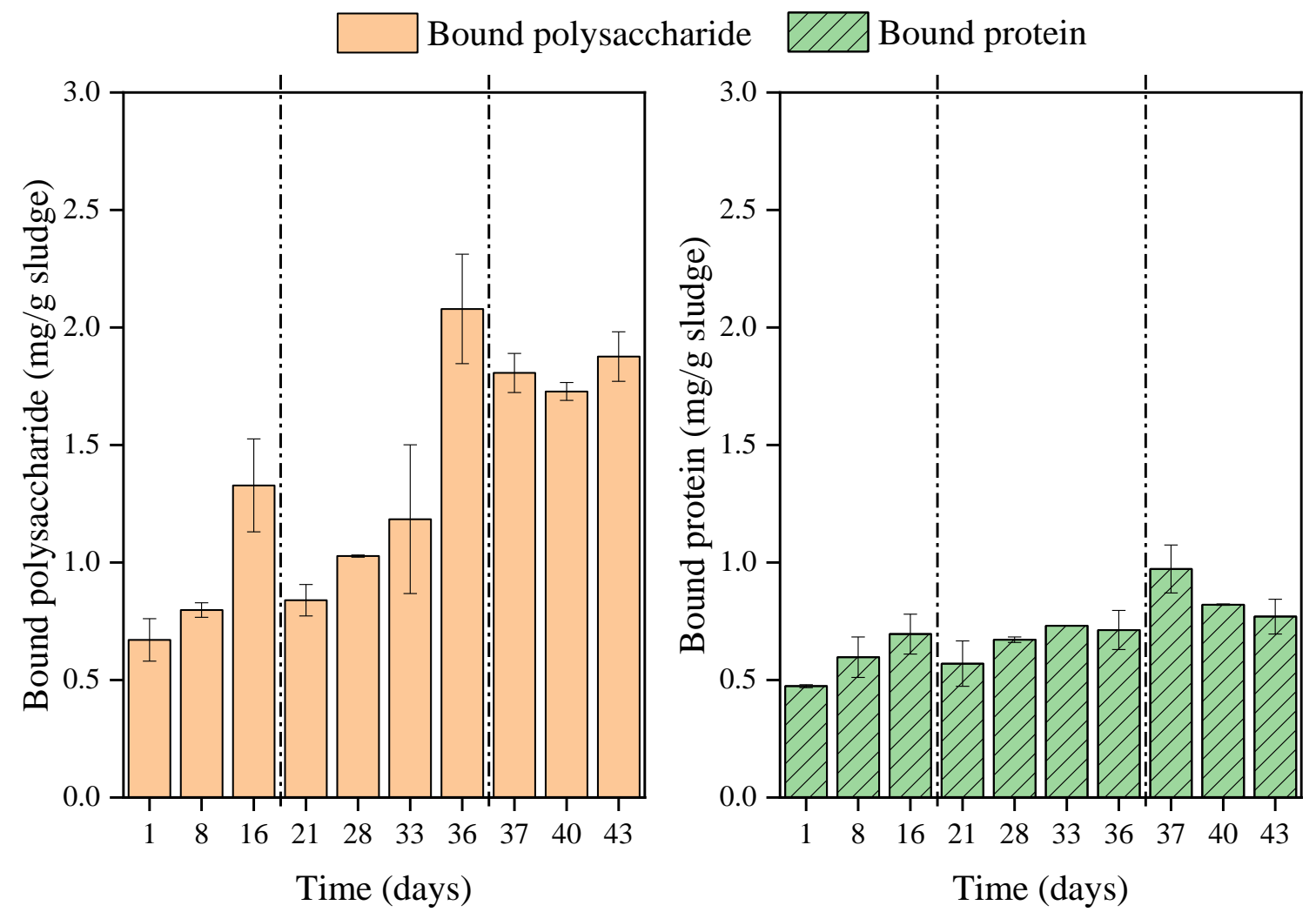

Figure S6. Extracellular polymeric substances (EPS) concentration in the mixed liquor during the experiment normalized to biomass concentration. The error bar represents the standard deviation from duplicate samples. 


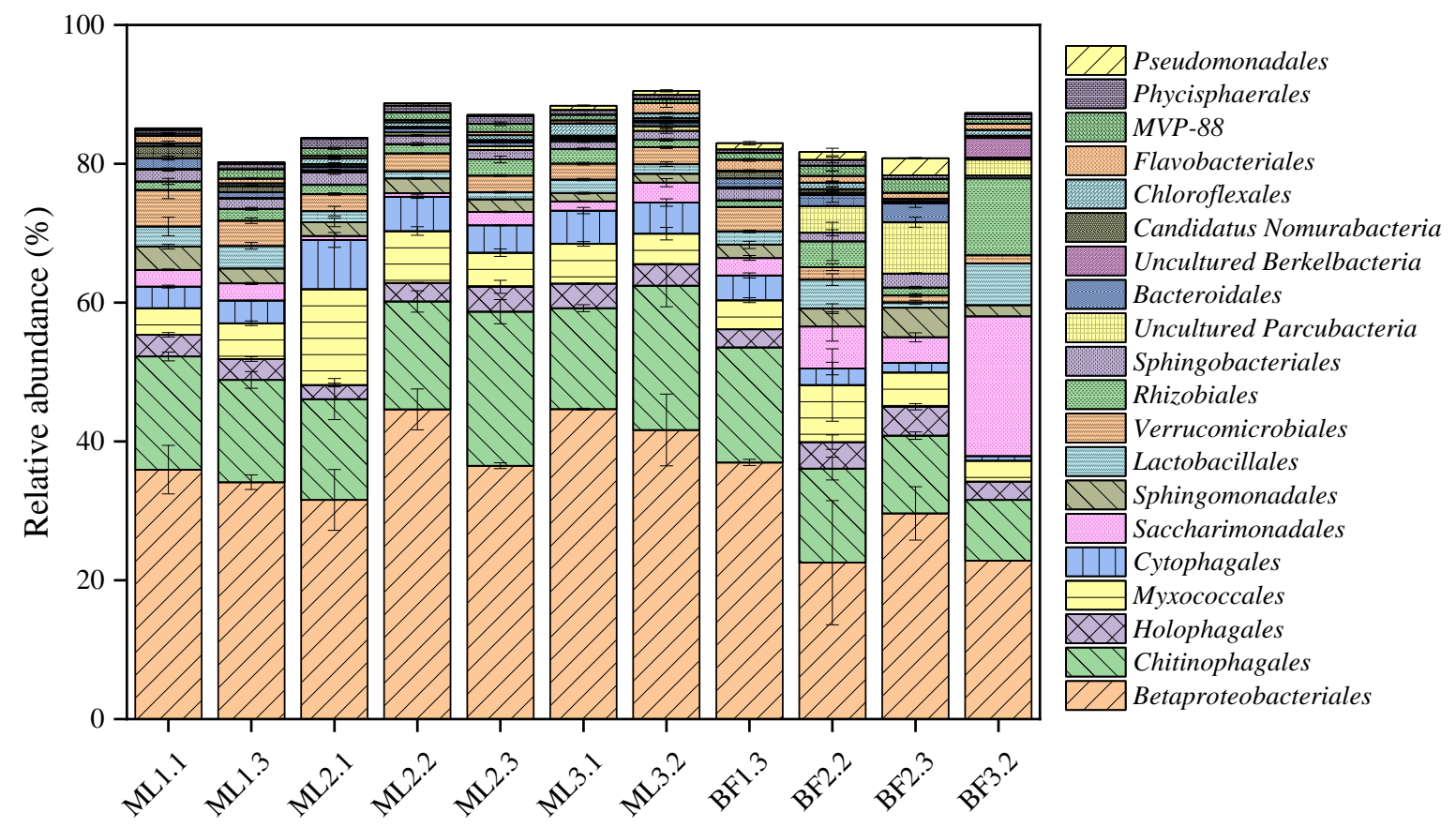

Sample

Figure S7. Relative abundances of top 10 orders in sample (a total of 20 orders) of mixed liquor (ML) and biofilm (BF). 
Table S1. Pearson correlation between microbial orders in the mixed liquor with fouling indicators. Taxa that were identified as network connectors/module hubs are marked with an asterisk. Taxa that showed strong correlations with fouling indicator $(>0.6)$ are marked with two asterisks.

\begin{tabular}{|c|c|c|c|c|}
\hline Taxa & SMPc & SMPp & EPSc & EPSp \\
\hline Absconditabacteriales SRI & 0.03 & 0.177 & 0.062 & 0 \\
\hline Acetobacterales & 0.001 & 0.452 & 0.212 & 0.179 \\
\hline Anaerolinea 1-20 & 0.025 & 0.36 & 0.113 & 0 \\
\hline Anaerolineales & 0.129 & 0.075 & 0.001 & 0.003 \\
\hline Armatimonadales & 0.489 & 0.446 & 0.27 & 0.139 \\
\hline Azospirillales & 0.585 & 0.214 & 0.011 & 0.103 \\
\hline Babeliales** & 0.615 & 0.118 & 0.032 & 0.021 \\
\hline Bacteroidales & 0.027 & 0.008 & 0.16 & 0.223 \\
\hline Bdellovibrionales & 0.359 & 0.047 & 0.152 & 0.176 \\
\hline Betaproteobacteriales & 0.016 & 0 & 0.002 & 0.026 \\
\hline Blastocatellales** & 0.736 & 0.136 & 0.056 & 0.004 \\
\hline Blastocatellia 11-24 & 0.084 & 0.212 & 0.23 & 0.128 \\
\hline Caedibacterales** & 0.906 & 0.588 & 0.072 & 0.284 \\
\hline Caldilineales & 0.071 & 0.065 & 0.115 & 0.033 \\
\hline Candidatus Falkowbacteria** & 0.135 & 0 & 0.908 & 0.769 \\
\hline Candidatus Magasanikbacteria & 0.074 & 0.146 & 0.15 & 0.088 \\
\hline Candidatus Nomurabacteria & 0.096 & 0.001 & 0.216 & 0.265 \\
\hline Candidatus Pacebacteria & 0.049 & 0.352 & 0.024 & 0.076 \\
\hline Caulobacterales & 0.073 & 0.055 & 0.216 & 0.187 \\
\hline CCM19a & 0.181 & 0.047 & 0.09 & 0.339 \\
\hline Chitinophagales & 0.007 & 0.455 & 0.26 & 0.114 \\
\hline Chlamydiales & 0.054 & 0.111 & 0.086 & 0.041 \\
\hline Chloroflexales & 0.057 & 0.004 & 0.004 & 0.059 \\
\hline Chthoniobacterales & 0 & 0 & 0.139 & 0.115 \\
\hline Chthonomonadales & 0.08 & 0.001 & 0.017 & 0.006 \\
\hline Clostridiales & 0.283 & 0.007 & 0.028 & 0.025 \\
\hline Cytophagales & 0.13 & 0 & 0.082 & 0.051 \\
\hline Desulfarculales & 0.47 & 0.291 & 0.034 & 0.166 \\
\hline Desulfobacterales & 0.015 & 0.335 & 0.093 & 0.008 \\
\hline Desulfovibrionales & 0.31 & 0.037 & 0.183 & 0.193 \\
\hline Desulfuromonadales & 0.2 & 0.008 & 0.001 & 0.001 \\
\hline Diplorickettsiales** & 0.767 & 0.255 & 0 & 0.033 \\
\hline Dongiales $^{* *}$ & 0.093 & 0.034 & 0.634 & 0.805 \\
\hline Erysipelotrichales** & 0.086 & 0.057 & 0.945 & 0.717 \\
\hline Fibrobacterales** & 0.002 & 0.741 & 0.26 & 0.085 \\
\hline Fimbriimonadales & 0.201 & 0.073 & 0.005 & 0.026 \\
\hline Flavobacteriales & 0.053 & 0.326 & 0.006 & 0 \\
\hline Frankiales & 0.065 & 0.004 & 0.15 & 0.139 \\
\hline Gammaproteobacteria Incertae Sedis & 0 & 0.025 & 0.062 & 0.09 \\
\hline Gemmatales & 0.033 & 0.038 & 0.377 & 0.281 \\
\hline Gemmatimonadales & 0.309 & 0.089 & 0.18 & 0.103 \\
\hline Holophagales & 0.096 & 0.075 & 0.039 & 0.034 \\
\hline
\end{tabular}




\begin{tabular}{|c|c|c|c|c|}
\hline Holosporales** & 0.682 & 0.007 & 0.372 & 0.399 \\
\hline Hydrogenedentiales & 0.318 & 0.161 & 0.17 & 0.024 \\
\hline Isosphaerales & 0.06 & 0.297 & 0.097 & 0.001 \\
\hline Lactobacillales & 0.338 & 0.041 & 0.129 & 0.062 \\
\hline Legionellales** & 0.506 & 0.351 & 0.993 & 0.768 \\
\hline Leptospirales & 0.054 & 0.002 & 0 & 0.021 \\
\hline Micrococcales & 0.149 & 0.196 & 0.006 & 0.009 \\
\hline Microtrichales & 0.383 & 0.448 & 0.475 & 0.384 \\
\hline$M V P-88$ & 0.148 & 0.269 & 0.018 & 0.044 \\
\hline Myxococcales & 0.035 & 0.049 & 0.228 & 0.196 \\
\hline$N B 1 j$ & 0.12 & 0.123 & 0.01 & 0 \\
\hline Nitrospirales & 0.002 & 0.117 & 0.056 & 0.128 \\
\hline Obscuribacterales & 0.106 & 0.002 & 0.198 & 0.273 \\
\hline Oligoflexales & 0.531 & 0.049 & 0.19 & 0.16 \\
\hline OPB56 & 0.023 & 0.005 & 0.243 & 0.28 \\
\hline Opitutales & 0.131 & 0.004 & 0.006 & 0.002 \\
\hline Paracaedibacterales $* *$ & 0.802 & 0.258 & 0.005 & 0.061 \\
\hline Pedosphaerales & 0.085 & 0.107 & 0.151 & 0.161 \\
\hline Phycisphaeraemle18 & 0.312 & 0.106 & 0 & 0.026 \\
\hline Phycisphaerales & 0.037 & 0.034 & 0.029 & 0.061 \\
\hline Pirellulales & 0.005 & 0.001 & 0.146 & 0.2 \\
\hline Planctomycetales & 0.035 & 0.002 & 0.316 & 0.398 \\
\hline Propionibacteriales & 0.023 & 0.006 & 0.294 & 0.252 \\
\hline Pseudomonadales & 0.002 & 0.07 & 0.087 & 0.162 \\
\hline RBG13549 & 0.111 & 0.003 & 0 & 0.007 \\
\hline Reyranellales & 0.294 & 0.178 & 0.095 & 0.079 \\
\hline Rhizobiales & 0 & 0.019 & 0.174 & 0.167 \\
\hline Rhodobacterales & 0.536 & 0.22 & 0.063 & 0.025 \\
\hline Rhodospirillales & 0 & 0.16 & 0.03 & 0.022 \\
\hline Rickettsiales** & 0.708 & 0.264 & 0.018 & 0 \\
\hline Saccharimonadales & 0.299 & 0.217 & 0.086 & 0.039 \\
\hline SAR324 clade Marine group $B$ & 0.027 & 0.057 & 0.079 & 0.157 \\
\hline SBR1031 & 0.024 & 0.097 & 0.489 & 0.483 \\
\hline Selenomonadales & 0.11 & 0.107 & 0.042 & 0.045 \\
\hline SM1A07 & 0 & 0.236 & 0.019 & 0.078 \\
\hline Solibacterales & 0.183 & 0.131 & 0.097 & 0.068 \\
\hline Sphingobacteriales & 0.014 & 0.015 & 0.188 & 0.292 \\
\hline Sphingomonadales & 0.011 & 0.009 & 0.279 & 0.372 \\
\hline Spirochaetales & 0.018 & 0.048 & 0 & 0.046 \\
\hline Steroidobacterales & 0.479 & 0.08 & 0.341 & 0.243 \\
\hline Tepidisphaerales & 0.389 & 0.002 & 0.014 & 0.004 \\
\hline Thermales & 0.377 & 0.322 & 0.022 & 0.004 \\
\hline Thermoanaerobaculales & 0.428 & 0.219 & 0.005 & 0.001 \\
\hline Thermomicrobiales & 0 & 0.278 & 0.218 & 0.174 \\
\hline Tistrellales & 0.161 & 0.278 & 0.205 & 0.119 \\
\hline Unassigned Acidobacteria & 0.165 & 0.133 & 0.005 & 0.03 \\
\hline
\end{tabular}




\begin{tabular}{|c|c|c|c|c|}
\hline Unassigned Alphaproteobacteria** & 0.778 & 0.223 & 0 & 0.028 \\
\hline Unassigned Bacteroidia & 0.016 & 0.185 & 0.182 & 0.2 \\
\hline Unassigned Chloroflexi & 0 & 0.1 & 0.2 & 0.077 \\
\hline Unassigned Deltaproteobacteria & 0.002 & 0.102 & 0.03 & 0.037 \\
\hline Unassigned Elusimicrobia & 0.076 & 0.18 & 0.013 & 0.014 \\
\hline Unassigned LineageIIa & 0.357 & 0.06 & 0.003 & 0.016 \\
\hline Unassigned Microgenomatia & 0.135 & 0.513 & 0.391 & 0.143 \\
\hline Unassigned microorganism & 0.337 & 0.525 & 0.005 & 0.024 \\
\hline Unassigned OM190 & 0.024 & 0.015 & 0.008 & 0.049 \\
\hline Unassigned Subgroup 17** & 0.537 & 0 & 0.592 & 0.653 \\
\hline Unassigned Subgroup 6 & 0.183 & 0.058 & 0.013 & 0.065 \\
\hline UnassignedBacteria & 0.044 & 0.135 & 0.313 & 0.498 \\
\hline Uncultured Alphaproteobacteria & 0.219 & 0.091 & 0.055 & 0.071 \\
\hline Uncultured Berkelbacteria* & 0.378 & 0.617 & 0.483 & 0.466 \\
\hline Uncultured LCP89** & 0.008 & 0 & 0.592 & 0.644 \\
\hline Uncultured OM190 & 0 & 0.09 & 0.04 & 0 \\
\hline Uncultured Parcubacteria* & 0.13 & 0.322 & 0.202 & 0.09 \\
\hline Uncultured Pelobacter sp. & 0.396 & 0.149 & 0.168 & 0.063 \\
\hline Uncultured Woesearchaeia & 0.338 & 0.065 & 0.15 & 0.081 \\
\hline Uncultured WS6 Dojkabacteria & 0.015 & 0.205 & 0.38 & 0.091 \\
\hline Verrucomicrobiales & 0.074 & 0 & 0.215 & 0.229 \\
\hline Victivallales* & 0.026 & 0.006 & 0.669 & 0.764 \\
\hline$W D 260 * *$ & 0.57 & 0.725 & 0.395 & 0.352 \\
\hline Xanthomonadales & 0.014 & 0.004 & 0.044 & 0.151 \\
\hline
\end{tabular}

Table S2. Pearson correlation between microbial orders in the biofilm with fouling indicators. Taxa that were identified as network connectors/module hubs are marked with an asterisk. Taxa that showed strong correlations with fouling indicator $(>0.6)$ are marked with two asterisks.

\begin{tabular}{|l|l|l|l|l|}
\hline Taxa & SMPc & SMPp & EPSc & EPSp \\
\hline Absconditabacteriales SR1** & 0.279 & 0.701 & 0.465 & 0.074 \\
\hline Acetobacterales & 0.163 & 0.25 & 0.516 & 0.116 \\
\hline Anaerolineales** & 0.221 & 0.06 & 0.491 & 0.928 \\
\hline Azospirillales** & 0.39 & 0.639 & 0.918 & 0.254 \\
\hline Babeliales & 0.102 & 0.002 & 0.093 & 0.027 \\
\hline Bacteroidales & 0.04 & 0.037 & 0.079 & 0.004 \\
\hline Bdellovibrionales & 0.515 & 0.202 & 0 & 0.006 \\
\hline Betaproteobacteriales & 0.165 & 0.074 & 0.024 & 0.015 \\
\hline Blastocatellales & 0.529 & 0.269 & 0.026 & 0.001 \\
\hline Blastocatellia 11-24* & 0.728 & 0.44 & 0.123 & 0.008 \\
\hline Brevinematales* & 0.075 & 0 & 0.316 & 0.8 \\
\hline Caedibacterales** & 0.936 & 0.153 & 0 & 0.042 \\
\hline Caldilineales & 0.104 & 0 & 0.095 & 0.023 \\
\hline $\begin{array}{l}\text { Candidate division WOR1 bacterium } \\
\text { RIFOXYB2FULL4235* }\end{array}$ Fandidatus Collierbacteria & 0.499 & 0.969 & 0.535 & 0.4 \\
\hline Candidatus Falkowbacteria & 0.039 & 0.297 & 0.346 & 0.204 \\
\hline
\end{tabular}




\begin{tabular}{|c|c|c|c|c|}
\hline Candidatus Nomurabacteria & 0.312 & 0.49 & 0.289 & 0.114 \\
\hline Candidatus Pacebacteria & 0 & 0.029 & 0.124 & 0.194 \\
\hline Candidatus Zambryskibacteria & 0.082 & 0.07 & 0.095 & 0.004 \\
\hline Caulobacterales $* *^{*}$ & 0.017 & 0.14 & 0.613 & 0.997 \\
\hline Chitinophagales & 0.164 & 0.484 & 0.182 & 0.172 \\
\hline Chlamydiales & 0.004 & 0.011 & 0 & 0.035 \\
\hline Chloroflexales & 0.449 & 0.004 & 0.255 & 0.408 \\
\hline Chthonomonadales $* *$ & 0.645 & 0.556 & 0.093 & 0.033 \\
\hline Clostridiales & 0.016 & 0.114 & 0.369 & 0.085 \\
\hline Coxiellales & 0.019 & 0.025 & 0.035 & 0.045 \\
\hline Cytophagales** & 0.343 & 0.628 & 0.245 & 0.099 \\
\hline Desulfarculales** & 0.579 & 0.199 & 0.78 & 0.54 \\
\hline Desulfobacterales & 0 & 0.197 & 0.26 & 0.011 \\
\hline Desulfovibrionales & 0.036 & 0.537 & 0.576 & 0.099 \\
\hline Desulfuromonadales & 0 & 0.436 & 0.064 & 0.02 \\
\hline Diplorickettsiales & 0.134 & 0.201 & 0.136 & 0.009 \\
\hline Fibrobacterales & 0.317 & 0.056 & 0.232 & 0.008 \\
\hline Fimbriimonadales & 0.451 & 0.412 & 0.016 & 0 \\
\hline Flavobacteriales & 0.14 & 0.195 & 0.088 & 0.093 \\
\hline Frankiales & 0.01 & 0.257 & 0.016 & 0.023 \\
\hline Gammaproteobacteria Incertae Sedis & 0.104 & 0.035 & 0.065 & 0.121 \\
\hline Gemmatales & 0.038 & 0.168 & 0.327 & 0.065 \\
\hline Gemmatimonadales & 0.537 & 0.445 & 0.122 & 0.004 \\
\hline Holophagales & 0.392 & 0.025 & 0.094 & 0 \\
\hline Holosporales & 0.278 & 0.551 & 0.248 & 0.126 \\
\hline Isosphaerales** & 0.135 & 0.259 & 0.677 & 0.163 \\
\hline Lactobacillales & 0.061 & 0.005 & 0.334 & 0.304 \\
\hline Leptospirales & 0.427 & 0.03 & 0.211 & 0.086 \\
\hline Micrococcales $* *$ & 0.002 & 0.215 & 0.632 & 0.327 \\
\hline$M V P-88$ & 0.123 & 0.036 & 0.129 & 0.002 \\
\hline Myxococcales & 0.126 & 0.071 & 0.098 & 0.12 \\
\hline$N B 1 j * *$ & 0.002 & 0.946 & 0.708 & 0.333 \\
\hline Nitrospirales** & 0.706 & 0.097 & 0.056 & 0.023 \\
\hline Obscuribacterales & 0.147 & 0.389 & 0.243 & 0.165 \\
\hline Oligoflexales & 0.016 & 0 & 0 & 0.035 \\
\hline OPB56 & 0.024 & 0.005 & 0.108 & 0.004 \\
\hline Opitutales & 0.328 & 0.033 & 0.075 & 0.085 \\
\hline Paracaedibacterales & 0.768 & 0.272 & 0.013 & 0.016 \\
\hline Pedosphaerales & 0.459 & 0.377 & 0.094 & 0.016 \\
\hline Phycisphaerales & 0.199 & 0.003 & 0.112 & 0.152 \\
\hline Pirellulales* & 0.013 & 0.011 & 0.169 & 0.038 \\
\hline Planctomycetales & 0.012 & 0.249 & 0.473 & 0.274 \\
\hline Pseudomonadales & 0.102 & 0.056 & 0.242 & 0.017 \\
\hline RBG13549 & 0.383 & 0.119 & 0.006 & 0.166 \\
\hline Reyranellales & 0.007 & 0.389 & 0.423 & 0.276 \\
\hline Rhizobiales & 0.075 & 0.134 & 0.014 & 0.034 \\
\hline Rhodobacterales & 0.006 & 0.134 & 0.102 & 0.062 \\
\hline Rhodospirillales & 0.232 & 0.2 & 0.059 & 0.034 \\
\hline
\end{tabular}




\begin{tabular}{|c|c|c|c|c|}
\hline Rickettsiales** & 0.704 & 0.2 & 0.002 & 0.013 \\
\hline Saccharimonadales & 0.064 & 0.303 & 0 & 0.011 \\
\hline SAR324 clade Marine group $B^{* *}$ & 0.1 & 0.636 & 0.352 & 0.015 \\
\hline SBR1031 & 0.109 & 0.004 & 0.321 & 0.526 \\
\hline Selenomonadales & 0.134 & 0.378 & 0.158 & 0.001 \\
\hline Solibacterales $* *$ & 0.798 & 0.378 & 0.041 & 0.012 \\
\hline Sphingobacteriales & 0.005 & 0.009 & 0.121 & 0.021 \\
\hline Sphingomonadales & 0.195 & 0.158 & 0.305 & 0.035 \\
\hline Spirochaetales $* *$ & 0.097 & 0.943 & 0.725 & 0.19 \\
\hline Steroidobacterales & 0.341 & 0.004 & 0.059 & 0.052 \\
\hline Synergistales** & 0.599 & 0.77 & 0.691 & 0.496 \\
\hline Tepidisphaerales* & 0.82 & 0.083 & 0.997 & 0.956 \\
\hline Thermales $* *$ & 0.704 & 0.174 & 0.01 & 0.104 \\
\hline Thermoanaerobaculales* & 0.589 & 0.629 & 0.291 & 0.035 \\
\hline Tistrellales & 0.151 & 0.002 & 0.188 & 0.151 \\
\hline Unassigned Acidobacteria & 0.325 & 0.01 & 0.204 & 0.155 \\
\hline Unassigned Alphaproteobacteria** & 0.889 & 0.206 & 0.01 & 0.019 \\
\hline Unassigned Bacteria** & 0.402 & 0.88 & 0.709 & 0.147 \\
\hline Unassigned Bacteroidia* & 0.173 & 0.325 & 0.495 & 0.509 \\
\hline Unassigned Chloroflexi & 0.314 & 0.023 & 0.038 & 0.01 \\
\hline Unassigned Deltaproteobacteria & 0.04 & 0.746 & 0.241 & 0.025 \\
\hline Unassigned LineageIIa & 0.371 & 0.271 & 0.545 & 0.344 \\
\hline Unassigned Margulisbacteria* & 0.435 & 0.976 & 0.856 & 0.512 \\
\hline Unassigned Microgenomatia** & 0.282 & 0.603 & 0.276 & 0.002 \\
\hline Unassigned microorganism $* *$ & 0.23 & 0.639 & 0.759 & 0.172 \\
\hline Unassigned OM190 & 0.228 & 0.119 & 0.155 & 0.112 \\
\hline Unassigned Parcubacteria*** & 0.626 & 0.164 & 0.018 & 0.268 \\
\hline Unassigned Subgroup 6 & 0.332 & 0.426 & 0.27 & 0.072 \\
\hline Unassigned WS6 Dojkabacteria & 0.597 & 0.561 & 0.111 & 0.124 \\
\hline Uncultured Alphaproteobacteria** & 0.686 & 0 & 0.227 & 0.175 \\
\hline Uncultured Berkelbacteria & 0.421 & 0.303 & 0.009 & 0.039 \\
\hline Uncultured OM190* & 0.083 & 0.222 & 0.633 & 0.311 \\
\hline Uncultured Parcubacteria & 0.421 & 0.272 & 0.212 & 0 \\
\hline Uncultured Woesearchaeia & 0.017 & 0.29 & 0.13 & 0 \\
\hline Uncultured WS6 Dojkabacteria & 0.345 & 0.084 & 0.014 & 0.046 \\
\hline Verrucomicrobiales & 0.583 & 0.566 & 0.163 & 0.007 \\
\hline Victivallales & 0.235 & 0.048 & 0.05 & 0.007 \\
\hline Xanthomonadales & 0.3 & 0.188 & 0.159 & 0.171 \\
\hline
\end{tabular}

